\title{
The meta-analysis of transcriptomes of Arabidopsis thaliana transgenic plants with altered expression of dual-targeting RNA-polymerase RPOTmp
}

\author{
Igor Vladimirovich Gorbenko \\ Laboratory of Plant Genetic \\ Engineering \\ SIPPB SB RAS \\ Irkutsk, Russia \\ gravov.chemistry@gmail.com
}

Vadim Igorevich Belkov

Laboratory of Plant Genetic

Engineering

SIPPB SB RAS

Irkutsk, Russia

anvad.irk@rambler.ru

\author{
Vladislav Igorevich Tarasenko \\ Laboratory of Plant Genetic \\ Engineering \\ SIPPB SB RAS \\ Irkutsk, Russia \\ vslav@inbox.ru
}

Yuri Mikhailovich Konstantinov

Laboratory of Plant Genetic

Engineering

SIPPB SB RAS

Irkutsk, Russia

yukon@sifibr.irk.ru

\author{
Alexander Igorevich Katyshev \\ Laboratory of Plant Physiological \\ Genetics \\ SIPPB SB RAS \\ Irkutsk, Russia \\ alex@sifibr.irk.ru
}

Milana Viacheslavovna Koulintchenko

Laboratory of Plant Genetic

Engineering

SIPPB SB RAS

Irkutsk, Russia

mk100171@yahoo.com

\begin{abstract}
In dicotyledons, RPOTmp is one of three phagetype RNA polymerases encoded in the nucleus and transported to both mitochondria and chloroplasts. It was established that the main functions of RPOTmp are associated, however, with mitochondria, and not with chloroplasts. The activity of RPOTmp in mitochondria is manifested throughout all stages of plant development, where together with the main RNA polymerase of these organelles RPOTm, it transcribes genes encoding subunits of the I and IV respiratory complexes, the protein synthesis apparatus, as well as a number of mitochondrial proteins with unknown functions. The impairment of RPOTmp functions at the molecular level leads to a significant decrease in the activity of $I$ and IV respiratory complexes, at the organism level, to a delay in germination, a number of phenotypic differences in adult plants and a significant delay in flowering. Many aspects of the organelle gene expression regulation, and in particular RPOTmp, are still unknown. The fact of dual localization indicates a possible role of this NEP in anterograde signaling. Comprehensive analysis of transcriptomes data of several transgenic lines obtained by DNA microarray method was done using the bioinformatic approach. Bioinformatic analysis of transcriptomic and metabolic changes in transgenic plants with altered RPOTmp expression is presented, the presumptive role of this enzyme in organelle gene expression regulation as well as in retrograde signaling regulation in plants is discussed.
\end{abstract}

Keywords - mitochondria, chloroplasts, Arabidopsis thaliana, transcriptome, gene expression regulation, RNAP, retrograde and anterograde regulation

\section{Introduction}

It is well-known that cell organelles produce ATP and a number of vital metabolites which causes their importance for plant growth and development. Despite the fact that most of organelle genome was transferred to nucleus during the evolutional process, mitochondria and chloroplasts saved their own genetic apparatus. Several thousand of proteins encoded in nuclear and organellar DNA define organelles structure and function and emphasize the significance of OGE (Organellar Gene Expression) and NGE (Nuclear Gene Expression) coordination. Up to date a significant bulk of data characterizing chloroplast gene transcription and regulatory pathways that control chloroplast function obtained. On the other hand, mitochondrial gene expression regulation involving nuclear-encoded transcription factors and various cell metabolites is still insufficiently explored in many aspects. We believe that the model developed in our laboratory using transgenic plants of Arabidopsis with complementation or overexpression of RPOTmp functions, one of the organellar NEP (Nuclear Encoded Polymerase), targeting either in mitochondria or in chloroplasts, will allow us to identify and study factors involved in the regulation of OGE, or indirectly, via expression regulation of gene encoding this NEP, in retrograde and anterograde signaling.

To investigate the character of transcriptome changes dependent on altered RPOTmp expression, we carried out DNA-microarray assays and performed bioinformatic analysis of obtained data.

\section{Results}

\section{Analysed transgenic plants}

Earlier, Arabidopsis thaliana transgenic plants with RPOTmp overexpression in mitochondria or chloroplasts were obtained in our laboratory (Tarasenko et al., 2016). Four sets of transgenic lines were obtained on the basis of the Arabidopsis wild type plants (Columbia- 0 ), (Col-M and Col$\mathrm{P}$ lines), and on the basis of the rpotmp mutant line with inactivated RPOT2 gene encoding RPOTmp, (Tmp-M and Tmp-P lines). The obtained transformants are characterized by cytoplasmic expression in their cells of recombinant RPOTmp containing a transit peptide for protein targeting to mitochondria (Col-M and Tmp-M lines) or to chloroplasts (lines Col-P and Tmp-P). The Col-M/Col-P lines and the Tmp-M/Tmp-P lines are characterized by RPOTmp overexpression or by complementation of RPOTmp functions respectively.

For DNA microarray analysis, total RNA was extracted from 12-days old seedlings of the wild type, the rpotmp mutant line, Col-M, Col-P, Tmp-M and Tmp-P lines. Extracted DNA microarray data were used to perform a highthroughput full-genome transcriptomic analysis of the studied lines in comparison with the wild-type plants. Three independent biological replicates were analyzed, each represented RNA preparations obtained from several plants of the studied lines. 


\section{Data obtained}

Using multiple comparison method, 2301 genes were estimated as differentially expressed with $\mathrm{p}<0.05$ and lfc $>1$ (all data was estimated and statistically tested using limma $\mathrm{R}$ package [1], the p-value of each gene was corrected by the Benjamini and Hochberg method). Among the DEG array, 61 records were found to be annotated as transcription factors (TF). There were found 10 ethylene response TFs, 9 TFs with the MADS-box domain, 7 TFs of the WRKY family, 6 TFs with the MYB domain and the bHLH motif. The presence of two heat shock factors was observed, HSFA6A, the expression of which was increased in Col-P12 line and in Tmp-M lines, and HSF30 with reduced expression in ColM20 and Col-P5 transgenic lines.

To deepen our understanding of cell transcriptome changes caused by modified mitochondrial and chloroplast expression of RPOTmp, we performed co-expression analysis and estimated co-expression modules. RPOTmp was found to be located in module 1. We used INTERACTOME 2.0 [2], a predicted protein-protein interactions database to construct protein-protein interactions network (fig. 1). We found that RPOTmp is probably connected via AT5G52640 (HSP81.1 / 83 / 90.1, cytosolic) with large network nodes: AT3G57150 (putative pseudouridine synthase (NAP57), nuclear and cytosolic), AT3G52140 (FMT, Friendly mitochondria) and AT4G37910 (mtHsc70-1, mitochondrial HSP70-1). HSP90.1 is a stress-induced (heat shock) holdase chaperone that is necessary for cell survival and besides is necessary for mediating brassinosteroid signals [3].

In order to find out which particular cellular processes are affected by modified expression of RPOTmp we carried out Gene Ontology and KEGG pathway analysis.

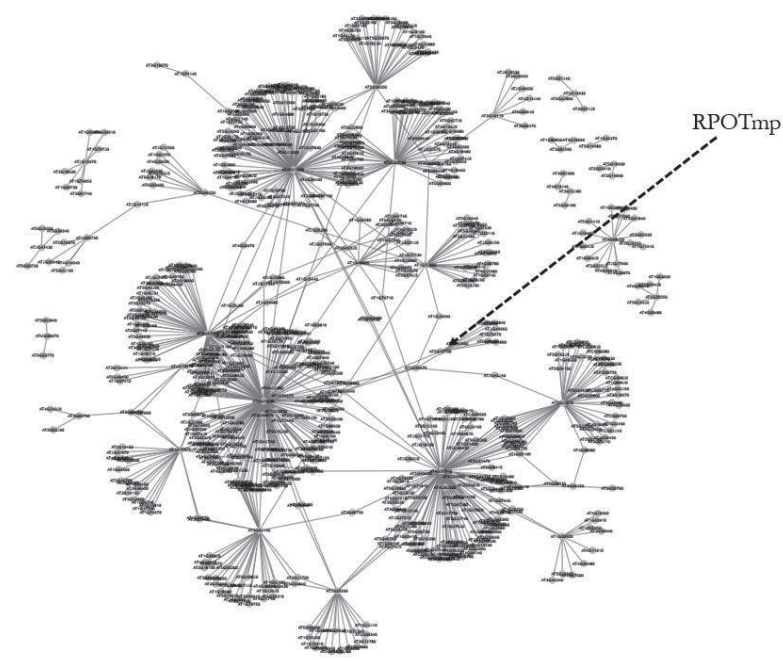

Fig. 1. Protein-protein interactions network of co-expression module I.

\section{Conclusions and perspectives}

In our study, it was found that the lack of functional RPOTmp in mitochondria (rpotmp and Tmp-P1 lines) caused a significant decrease in HSP90.1 expression, suggesting that these lines may have hypersensitivity to BRs. In the presence of RPOTmp in mitochondria at any level, the expression of HSP90.1 in our samples did not differ from the wild type.

Revealed by co-expression analysis, NAP57 and FMT communicate through a very large number of auxiliary proteins with one of the largest network node, fibrillarin 2 (FIB2, AT4G25630), a key nucleolar protein in eukaryotes that binds to the C / D box of small nucleolar RNAs (snoRNAs), directing methylation of 2'-ribose in rRNA and acts as a bridge for the transfer of information from genespecific regulatory proteins to the RNA polymerase II transcription apparatus $[4,5]$. Expression of FIB2 was found to be reduced (lfc $=-0.95, \mathrm{p}=0.018$ ) in Col-M15, the line with mitochondrial overexpression of RPOTmp.

According to Gene Ontology and KEGG pathway analysis, it was found that the Col-M15 line might have reduced rate of ribosome biogenesis. On the other hand, the rpotmp line lacking functional RPOTmp has reduced expression of genes that belong to plant-pathogen interactions pathway and increased expression of endocytosis-associated genes.

\section{References}

[1] B. Phipson, S. Lee, I. J. Majewski, W. S. Alexander, G. K. Smyth, "Robust hyperparameter estimation protects against hypervariable genes and improves power to detect differential expression", Ann Appl Stat, vol. 10, pp. 946-963, July 2016.

[2] J. Geisler-Lee, N. O'Toole, R. Ammar, N. J. Provart, A. H. Millar, M. Geisler, "A Predicted Interactome for Arabidopsis", Plant Physiology, vol. 145, pp. 317-329, October 2007.

[3] D. Samakovli, T. Margaritopoulou, C. Prassinos, D. Milioni, P. Hatzopoulo, "Brassinosteroid nuclear signaling recruits HSP90 activity", New Phytol, vol. 203, pp. 743-757, 2014.

[4] F. Barneche, F. Steinmetz, M. Echeverría, "Fibrillarin Genes Encode Both a Conserved Nucleolar Protein and a Novel Small Nucleolar RNA Involved in Ribosomal RNA Methylation in Arabidopsis thaliana", Journal of Biological Chemistry, vol. 275, pp. 27212-27220, 2000.

[5] D. Rakitina, M. Taliansky, J. Brown, N. Kalinina, "Two RNA-binding sites in plant fibrillarin provide interactions with variousRNA substrates", Nucleic acids research, vol. 39, pp. 8869-8880, 2011. 